\title{
Basic science: (July 2008)
}

1. Akil N, Yasmeen A, Kassab A, Ghabreau L, Darnel AD, Al Moustafa AE. High-risk human papillomavirus infections in breast cancer in Syrian women and their association with Id-1 expression: a tissue microarray study. $\mathrm{Br} J$ Cancer 2008; 99: 404-407.

2. Anders CK, Hsu DS, Broadwater G, Acharya CR, Foekens JA, Zhang Y, Wang YX, Marcom PK, Marks JR, Febbo PG, Nevins JR, Potti A, Blackwell KL. Young age at diagnosis correlates with worse prognosis and defines a subset of breast cancers with shared patterns of gene expression. J Clin Oncol 2008; 26: 3324-3330.

3. Barnett GC, Shah M, Redman K, Easton DF, Ponder BAJ, Pharoah PDP. Risk factors for the incidence of breast cancer: do they affect survival from the disease? J Clin Oncol 2008; 26: 3310-3316.

4. Bauerschmitz GJ, Ranki T, Kangasniemi L, Ribacka C, Eriksson M, Porten M, Herrmann I, Ristimaki A, Virkkunen P, Tarkkanen M, Hakkarainen T, Kanerva A, Rein D, Pesonen $\mathrm{S}$, Hemminki A. Tissue-specific promoters active in CD44(+)CD24(-/low) breast cancer cells. Cancer Res 2008; 68: 5533-5539.

5. Belkhiri A, Dar AA, Peng D, Razvi MH, Rinehart $C$, Arteaga CL, El-Rifai W. Expression of t-DARPP mediates trastuzumab resistance in breast cancer cells. Clin Cancer Res 2008; 14: 4564-4571.

6. Crandall CJ, Guan M, Laughlin GA, Ursin GA, Stanczyk FZ, Ingles SA, Barrett-Connor E, Greendale GA. Increases in serum estrone sulfate level are associated with increased mammographic density during menopausal hormone therapy. Cancer Epidemiol Biomarkers Prev 2008; 17: 1674-1681.

7. Diergaarde B, Potter JD, Jupe ER, Manjeshwar $S$, Shimasaki CD, Pugh TW, DeFreese DC, Gramling BA, Evans I, White E. Polymorphisms in genes involved in sex hormone metabolism, estrogen plus progestin hormone therapy use,

BCO/837/2008/JW and risk of postmenopausal breast cancer. Cancer Epidemiol Biomarkers Prev 2008; 17: 1751-1759.

8. Elsheikh SE, Green AR, Rakha EA, Samaka RM, Ammar AA, Powe D, Reis-Filho JS, Ellis IO. Caveolin 1 and caveolin 2 are associated with breast cancer basal-like and triple-negative immunophenotype. $\mathrm{Br} J$ Cancer 2008; 99: 327-334.

9. Fagerholm R, Hofstetter B, Tommiska J, Aaltonen K, Vrtel R, Syrjakoski K, Kallioniemi A, Kilpivaara O, Mannermaa A, Kosma VM, Uusitupa M, Eskelinen M, Kataja V, Aittomakii K, von Smitten K, Heikkila P, Lukas J, Holli K, Bartkova J, Blomqvist C, Bartek J, Nevanlinna $\mathrm{H}$. $\quad \mathrm{NAD}(\mathrm{P}) \mathrm{H}$ : quinone oxidoreductase 1 NQO1(star)2 genotype (P187S) is a strong prognostic and predictive factor in breast cancer. Nat Genet 2008; 40: 844-853.

10. Fleury L, Gerus M, Lavigne AC, Richard-Foy H, Bystricky K. Eliminating epigenetic barriers induces transient hormone-regulated gene expression in estrogen receptor negative breast cancer cells. Oncogene 2008; 27: 4075-4085.

11. Gail MH. Discriminatory accuracy from singlenucleotide polymorphisms in models to predict breast cancer risk. J Natl Cancer Inst 2008; 100: 1037-1041.

12. Ghoussaini $M$, Song HL, Koessler T, Al Olama AA, Kote-Jarai Z, Driver KE, Pooley KA, Ramus SJ, Kjaer SK, Hogdall E, DiCioccio RA, Whittemore AS, Gayther SA, Giles GG, Guy M, Edwards SM, Morrison J, Donovan JL, Hamdy FC, Dearnaley DP, Ardern-Jones AT, Hall AL, O'Brien LT, Gehr-Swain BN, Wilkinson RA, Brown PM, Hopper JL, Neal DE, Pharoah PDP, Ponder BAJ, Eeles RA, Easton DF, Dunning AM. Multiple loci with different cancer specificities within the 8q24 gene desert. $J$ Natl Cancer Inst 2008; 100: 962-966.

13. Golouh R, Cufer T, Sadikov A, Nussdorfer P, Usher PA, Brunner N, Schmitt M, Lesche R, Maier S, Timmermans M, Foekens JA, Martens JWM. The prognostic value of Stathmin-1, 
S100A2, and SYK proteins in ER-positive primary breast cancer patients treated with adjuvant tamoxifen monotherapy: an immunohistochemical study. Breast Cancer Res Treat 2008; 110: 317-326.

14. Hayes MJ, Thomas D, Emmons A, Giordano TJ, Kleer CG. Genetic changes of Wnt pathway genes are common events in metaplastic carcinomas of the breast. Clin Cancer Res 2008; 14: 4038-4044.

15. lakovlev VV, Arneson NCR, Wong V, Wang CJ, Leung S, lakovleva G, Warren K, Pintilie M, Done SJ. Genomic differences between pure ductal carcinoma in situ of the breast and that associated with invasive disease: a calibrated aCGH study. Clin Cancer Res 2008; 14: 4446-4454.

16. Jiang $W Q$, Zhu ZJ, Thompson HJ. Dietary energy restriction modulates the activity of AMP-activated protein kinase, Akt, and mammalian target of rapamycin in mammary carcinomas, mammary gland, and liver. Cancer Res 2008; 68: 5492-5499.

17. Kondo N, Toyama T, Sugiura H, Fujii $\mathrm{Y}$, Yamashita H. miR-206 expression is downregulated in estrogen receptor $\alpha$-positive human breast cancer. Cancer Res 2008; 68: 5004-5008.

18. Kovalchuk O, Filkowski J, Meservy J, linytskyy Y, Tryndyak VP, Chekhun VF, Pogribny IP. Involvement of microRNA-451 in resistance of the MCF-7 breast cancer cells to chemotherapeutic drug doxorubicin. Mol Cancer Ther 2008; 7: 2152-2159.

19. Lee CW, Raskett CM, Prudovsky I, Altieri DC. Molecular dependence of estrogen receptornegative breast cancer on a Notch-survivin signaling axis. Cancer Res 2008; 68: 5273-5281.

20. Lee-Hoeflich ST, Crocker L, Yao E, Pham T, Munroe X, Hoeflich KP, Sliwkowski MX, Stern HM. A central role for HER3 in HER2-amplified breast cancer: implications for targeted therapy. Cancer Res 2008; 68: 5878-5887.

21. Lord SJ, Bernstein L, Johnson KA, Malone KE, McDonald JA, Marchbanks PA, Simon MS, Strom BL, Press MF, Folger SG, Burkman RT, Deapen D, Spirtas R, Ursin G. Breast cancer risk and hormone receptor status in older women by parity, age of first birth, and breastfeeding: a case-control study. Cancer Epidemiol Biomarkers Prev 2008; 17: 1723-1730.

22. Nam DH, Jeon HM, Kim S, Kim MH, Lee YJ, Lee MS, Kim H, Joo KM, Lee DS, Price JE, Bang SI, Park WY. Activation of Notch signaling in a xenograft model of brain metastasis. Clin Cancer Res 2008; 14: 4059-4066.

23. O'Brien C, Cavet G, Pandita A, Hu XL, Haydu L, Mohan S, Toy K, Rivers CS, Modrusan Z, Amler LC, Lackner MR. Functional genomics identifies $A B C C 3$ as a mediator of taxane resistance in HER2-amplified breast cancer. Cancer Res 2008; 68: 5380-5389.

24. Rizzo P, Miao H, D'Souza G, Osipo C, Yun J, Zhao HP, Mascarenhas J, Wyatt D, Antico G, Hao L, Yao K, Rajan P, Hicks C, Siziopikou K, Selvaggi S, Bashir A, Bhandari D, Marchese A, Lendahl U, Qin JZ, Tonetti DA, Albain K, Nickoloff BJ, Miele L. Cross-talk between Notch and the estrogen receptor in breast cancer suggests novel therapeutic approaches. Cancer Res 2008; 68: 5226-5235.

25. Schmidt M, Bohm D, von Torne C, Steiner E, Puhl A, Pilch H, Lehr HA, Hengstler JG, Kolbl H, Gehrmann M. The humoral immune system has a key prognostic impact in node-negative breast cancer. Cancer Res 2008; 68: 5405-5413.

26. Steelman LS, Navolanic PM, Sokolosky ML, Taylor JR, Lehmann BD, Chappell WH, Abrams SL, Wong EWT, Stadelman KM, Terrian DM, Leslie NR, Martelli AM, Stivala F, Libra M, Franklin RA, McCubrey JA. Suppression of PTEN function increases breast cancer chemotherapeutic drug resistance while conferring sensitivity to mTOR inhibitors. Oncogene 2008; 27: 4086-4095.

27. Vasiljeva $O$, Korovin M, Gajda M, Brodoefel $H$, Bojic L, Kruger A, Schurigt U, Sevenich L, Turk B, Peters C, Reinheckel T. Reduced tumour cell proliferation and delayed development of highgrade mammary carcinomas in cathepsin Bdeficient mice. Oncogene 2008; 27: 4191-4199.

28. Wang $Y$, Dean JL, Millar EKA, Tran TH, McNeil CM, Burd CJ, Henshall SM, Utama FE, Witkiewicz A, Rui $H$, Sutherland RL, Knudsen KE, Knudsen ES. Cyclin D1b is aberrantly regulated in response to therapeutic challenge and promotes resistance to estrogen antagonists. Cancer Res 2008; 68: 5628-5638.

29. Zhang D, Shimizu T, Araki N, Hirota T, Yoshie M, Ogawa K, Nakagata N, Takeya M, Saya H. Aurora A overexpression induces cellular senescence in mammary gland hyperplastic tumors developed in p53-deficient mice. Oncogene 2008; 27: 4305-4314.

Prepared by

$R$ Sutherland

Cancer Research Program

Garvan Institute of Medical Research Darlinghurst, NSW, Australia 